\title{
PLANE CURVES CONTAINING A STAR CONFIGURATION
}

\author{
ENRICO CARLINI, ELENA GUARDO, AND ADAM VAN TUYL
}

\begin{abstract}
Given a collection of $l$ general lines $\ell_{1}, \ldots, \ell_{l}$ in $\mathbb{P}^{2}$, the star configuration $\mathbb{X}(l)$ is the set of points constructed from all pairwise intersections of these lines. For each non-negative integer $d$, we compute the dimension of the family of curves of degree $d$ that contain a star configuration.
\end{abstract}

\section{INTRODUCTION}

Throughout this paper, $S=k\left[x_{0}, x_{1}, x_{2}\right]$ with $k$ an algebraically closed field. Given any linear form $L \in S$, we let $\ell$ denote the corresponding line in $\mathbb{P}^{2}$. Let $\ell_{1}, \ldots, \ell_{l}$ be a set of $l$ distinct lines in $\mathbb{P}^{2}$. Classically, the union of these $l$ lines is called an l-lateral. A complete $l$-lateral is the union of $l$ lines, such that $\ell_{i} \cap \ell_{j} \cap \ell_{k}=\emptyset$ for all triples $\{i, j, k\} \subseteq\{1, \ldots, l\}$. We say that a plane curve has an inscribed $l$-lateral if it contains the $\left(\begin{array}{l}l \\ 2\end{array}\right)$ vertices of the $l$-lateral, that is, the $\left(\begin{array}{l}l \\ 2\end{array}\right)$ points formed by taking all possible intersections of lines.

Let $\mathbb{X}(l)$ denote the collection of points formed by taking all possible intersections of a complete l-lateral. Such a collection of points is sometimes called a star configuration. The name star configuration arises from the fact that a complete 5-lateral that contains an $\mathbb{X}(5)$ resembles a star. These special configurations, and their generalizations in $\mathbb{P}^{n}$, have recently risen in prominence due, in part, to the fact that they have nice algebraic properties (e.g., the minimal generators are products of linear forms), but at the same time exhibit some extremal properties (e.g., the work of Bocci and Harbourne [4] which compares symbolic and regular powers of ideals). The papers 11, 3, 10, 11, 12, 17, 18, are some of the papers that have contributed to our understanding of star configurations. Because this paper is related to our previous papers (see [5, 6]) on star configurations, we shall prefer to use the terminology of star configurations as opposed to the language of l-laterals found in [8, 15, 16. Moreover, star configurations may better lend themselves to higher dimensional generalizations (see our concluding remarks). Moving forward, we will primarily refer to the family of curves in $\mathbb{P}^{2}$ of degree $d$ that "contain a star configuration $\mathbb{X}(l)$ " as opposed to "contain an inscribed $l$-lateral".

In this paper we compute the dimension of the family of curves in $\mathbb{P}^{2}$ of degree $d$ that contain a star configuration $\mathbb{X}(l)$, or equivalently, an inscribed l-lateral. More precisely, if $l>2$, consider the quasi-projective variety

$$
\mathcal{D}_{l} \subseteq \underbrace{\check{\mathbb{P}}^{2} \times \cdots \times \check{\mathbb{P}}^{2}}_{l}
$$

2000 Mathematics Subject Classification. 14M05, 14H50.

Key words and phrases. star configuration points, plane curves. 
where $\left(\ell_{1}, \ldots, \ell_{l}\right) \in \mathcal{D}_{l}$ if and only if no three of the lines meet at a point; here $\check{\mathbb{P}}^{2}$ denotes the dual projective space. Notice that $\mathcal{D}_{l}$ can be seen as a parameter space for star configuration set of points obtained by intersecting $l$ general lines. With a slight abuse of notation, we will often write $\mathbb{X}(l) \in \mathcal{D}_{l}$, thus identifying a star configuration with the unique set of lines defining it.

We construct the following incidence correspondence

$$
\Sigma_{d, l}=\{(\mathcal{C}, \mathbb{X}(l)): \mathcal{C} \supseteq \mathbb{X}(l)\} \subseteq \mathbb{P} S_{d} \times \mathcal{D}_{l}
$$

Letting $\phi_{d, l}: \Sigma_{d, l} \rightarrow \mathbb{P} S_{d}$ denote the natural projection map, we define the locus of degree $d$ curves containing a star configuration $\mathbb{X}(l)$, denoted $\mathcal{S}(d, l)$, to be $\mathcal{S}(d, l)=\phi_{d, l}\left(\Sigma_{d, l}\right)$. We then prove the following result about the dimension of the locus.

Theorem 1.1. Let $d \geq 0$ and $l \geq 2$ be integers. Then $\mathcal{S}(d, l)=\emptyset$ if $d<l-1$, and

$$
\operatorname{dim} \mathcal{S}(d, l)= \begin{cases}\left(\begin{array}{c}
d+2 \\
d_{2}
\end{array}\right)-1 & \text { if } d \geq l-1 \text { and } l=2,3,4 \\
\left(\begin{array}{c}
d+2 \\
2
\end{array}\right)-2 & \text { if } d=4 \text { and } l=5 \\
\left(\begin{array}{c}
d+2 \\
2
\end{array}\right)-1 & \text { if } d \geq 5 \text { and } l=5 \\
\left(\begin{array}{c}
d+2 \\
2
\end{array}\right)-\left(\begin{array}{l}
l \\
2
\end{array}\right)+2 l-1 & \text { if } d \geq l-1 \text { and } l \geq 6 .\end{cases}
$$

Theorem 1.1 complements our previous work [5, 6] which showed that the generic degree $d$ plane curve contains a star configuration $\mathbb{X}(l)$ if and only if the projection map $\phi_{d, l}$ is dominant which happens if and only if $\operatorname{dim} \mathcal{S}(d, l)=\left(\begin{array}{c}d+2 \\ 2\end{array}\right)-1$. The tuples $(d, l)$ for which $\operatorname{dim} \mathcal{S}(d, l)=\left(\begin{array}{c}d+2 \\ 2\end{array}\right)-1$ are therefore precisely the tuples described in [6, Theorem 6.3]. The fact that $\mathcal{S}(d, l)=\emptyset$ for $d<l-1$ comes from Bezout's Theorem (see Remark 2.2).

It therefore suffices to focus on proving Theorem 1.1 for the pairs $(4,5)$ and $(d, l)$ with $d \geq l-1$ and $l \geq 6$. Our strategy for the pairs $(d, l) \neq(4,5)$ is to first translate the problem into computing the dimension of a graded ideal constructed from the linear forms $L_{1}, \ldots, L_{l}$ in a particular degree. This enables us to reduce the problem to computing the rank of a particular matrix. We use the notion of Lüroth quartics to deal with the pair $(d, l)=(4,5)$. Note that $\mathcal{S}(4,5)$ is the only $\mathcal{S}(d, l)$ whose dimension differs from the expected one.

The family $\mathcal{S}(d, d+1)$ was also studied by Barth [2, Application 2], who also computed their dimensions, and by Ellingsrud, Le Potier, and Strømme [9, Section 4], who raised the still-open question of computing the cardinality of the fibres of $\phi_{d, d+1}$.

Our paper is structured as follows. In Section 2 we recall the relevant facts about star configurations. We also translate our problem into a new algebraic question, and we compute $\operatorname{dim} \mathcal{S}(4,5)$. In Section 3 we prove Theorem 1.1 for all tuples $(d, 6)$ with $d \geq 5$. The results of this section provide a base case for the arguments of Section 4 . We conclude with remarks about the higher dimensional analog of this problem.

Acknowledgements The computer algebra system CoCoA [7] played an integral role in this project. The first author thanks the Università di Catania for its hospitality and the Politecnico di Torino for financial support. The third author acknowledges the support of NSERC. We would like to thank G. Ottaviani for suggesting this problem to us. We also thank the referee for his/her useful suggestions and improvements. 


\section{Properties of Star CONFIGURATions}

We recall the relevant results about star configurations in $\mathbb{P}^{2}$ and prove an upper bound on $\operatorname{dim} \mathcal{S}(d, l)$. Although some proofs are omitted, they can be found in [ㅍ, 11].

We continue to use the notation introduced in the introduction. For any $l \geq 2$, let $L_{1}, \ldots, L_{l}$ be a collection of $l$ linear forms of $S=k\left[x_{0}, x_{1}, x_{2}\right]$ that are three-wise linearly independent. We call such a collection a collection of general linear forms. We let $\mathbb{X}(l)$ denote the star configuration of $\left(\begin{array}{l}l \\ 2\end{array}\right)$ points in $\mathbb{P}^{2}$ which is formed from all pairwise intersections of the $l$ linear forms. Note that when $l=2$, then $\mathbb{X}(2)$ is simply a point, and if $l=3$, then $\mathbb{X}(3)$ is three non-collinear points.

The following lemma allows us to describe the minimal generators of the ideal associated to $\mathbb{X}(l)$ and the Hilbert function of this ideal.

Lemma 2.1. Let $L_{1}, \ldots, L_{l}$ be $l \geq 2$ general linear forms of $S=k\left[x_{0}, x_{1}, x_{2}\right]$, and let $I_{\mathbb{X}(l)}$ denote the defining ideal of $\mathbb{X}(l)$.

(i) For each $i \in\{1, \ldots, l\}$, let $\hat{L}_{i}=\prod_{j \neq i} L_{j}$. Then $I_{\mathbb{X}(l)}=\left(\hat{L}_{1}, \hat{L}_{2}, \ldots, \hat{L}_{l}\right)$.

(ii) The set of points $\mathbb{X}(l)$ has the Hilbert function of $\left(\begin{array}{l}l \\ 2\end{array}\right)$ generic points, that is

$$
H F(\mathbb{X}(l), t)=\operatorname{dim}_{k}\left(S / I_{\mathbb{X}(l)}\right)_{t}=\min \left\{\left(\begin{array}{c}
t+2 \\
2
\end{array}\right),\left(\begin{array}{l}
l \\
2
\end{array}\right)\right\} \text { for } t \geq 0 .
$$

Proof. For (i), see [5, Lemma 2.3(iv)]. For $(i i)$, see [5, Theorem 2.5].

Remark 2.2. Lemma $2.1(i)$ shows that no plane curve of degree $d$ with $d<l-1$ can contain a star configuration $\mathbb{X}(l)$. As a consequence, $\mathcal{S}(d, l)=\emptyset$ for all $(d, l)$ with $d<l-1$. Alternatively, one could use Bezout's theorem: if a degree $d<l-1$ curve is in $\mathcal{S}(d, l)$, every line of the star configuration meets the curve at the $(l-1)$ points obtained by the intersection of this line with the other $(l-1)$ lines, hence it is a component of the curve. A degree $d$ curve cannot contain $l>d+1$ lines, hence the result.

Remark 2.3. Tohăneanu [19] also considers ideals generated by products of linear forms with a connection to coding theory. When the linear forms are general, his results give an alternative proof to Lemma 2.1

We give an upper bound on $\operatorname{dim} \mathcal{S}(d, l)$.

Lemma 2.4. Let $l \geq 2$ and $d \geq l-1$ be integers. Then

$$
\operatorname{dim} \mathcal{S}(d, l) \leq\left(\begin{array}{c}
d+2 \\
2
\end{array}\right)-\left(\begin{array}{l}
l \\
2
\end{array}\right)+2 l-1
$$

Proof. Consider the incidence correspondence $\Sigma_{d, l}$ as given in (因), and let

$$
\psi_{d, l}: \Sigma_{d, l} \longrightarrow \mathcal{D}_{l} \text { and } \phi_{d, l}: \Sigma_{d, l} \longrightarrow \mathbb{P} S_{d}
$$

be the natural projection maps. Note that we are following the standard convention that $\mathbb{P} S_{d}$ is identified with the projective space $\mathbb{P}^{N_{d}}$ where $N_{d}=\left(\begin{array}{c}d+2 \\ 2\end{array}\right)-1$. Using a standard fibre dimension argument, if $d \geq l-1$, then

$\operatorname{dim} \Sigma_{d, l} \leq \operatorname{dim} \mathcal{D}_{l}+\operatorname{dim}_{k}\left(I_{\mathbb{X}(l)}\right)_{d}-1=\operatorname{dim} \mathcal{D}_{l}+\left(\begin{array}{c}2+d \\ d\end{array}\right)-\left(\begin{array}{l}l \\ 2\end{array}\right)-1=2 l+\left(\begin{array}{c}2+d \\ d\end{array}\right)-\left(\begin{array}{l}l \\ 2\end{array}\right)-1$ 
Here, we are using Lemma $2.1(i i)$ since $\operatorname{dim}_{k} S_{d}-\operatorname{dim}_{k}\left(I_{\mathbb{X}(l)}\right)_{d}=\left(\begin{array}{l}l \\ 2\end{array}\right)$ when $d \geq l-1$. The desired bound now follows from the fact that $\operatorname{dim} \mathcal{S}(d, l) \leq \operatorname{dim} \Sigma_{d, l}$.

Remark 2.5. In [2], Section 5, Lemma 2 or in [8], Lemma 6.3.24, the authors compute the dimension of $\left(I_{\mathbb{X}(l)}\right)_{l+1}$ and this result can be used to alternatively prove Lemma 2.4.

Remark 2.6. As shown in [6, Theorem 3.1], if $l \geq 6$, then the map $\phi_{d, l}$ cannot be dominant.

Inspired by our previous work [5, 6], we can reformulate the problem of computing $\operatorname{dim} \mathcal{S}(d, l)$ in terms of computing the dimension of an ideal in a specific degree. In fact, the proof of [6, Lemma 4.3] already contains the result we need. We first perform the following geometric construction. With $d \geq l-1$ define a map of affine varieties

$$
\Phi_{d, l}: \underbrace{S_{1} \times \cdots \times S_{1}}_{l} \times \underbrace{S_{d-l+1} \times \cdots \times S_{d-l+1}}_{l} \longrightarrow S_{d}
$$

such that

$$
\Phi_{d, l}\left(L_{1}, \ldots, L_{l}, M_{1}, \ldots, M_{l}\right)=\sum_{i=1}^{l} M_{i} \hat{L}_{i}
$$

We then rephrase our problem in terms of the map $\Phi_{d, l}$.

Lemma 2.7. With notation as above, the image of $\Phi_{d, l}$ is the affine cone over $\mathcal{S}(d, l)$. In particular, $\operatorname{dim} \mathcal{S}(d, l)=\operatorname{dim} \operatorname{Im}\left(\Phi_{d, l}\right)-1$.

Proof. Suppose $H$ is a degree $d$ form that defines a curve $\mathcal{C}$ that contains a star configuration $\mathbb{X}(l)$. So, there exists linear forms $L_{1}, \ldots, L_{l}$ such that $H \in\left(\hat{L}_{1}, \ldots, \hat{L}_{l}\right)$, and hence $H=\sum_{i=1}^{l} M_{i} \hat{L}_{i}$ with $M_{i} \in S_{d-l+1}$ for each $i$. But this means that $H \in \operatorname{Im}\left(\Phi_{d, l}\right)$. Viewing elements of $\mathcal{S}(d, l)$ as elements of $\mathbb{P} S_{d}$, this gives $\operatorname{dim} \mathcal{S}(d, l) \leq \operatorname{dim} \operatorname{Im}\left(\Phi_{d, l}\right)-1$.

Now consider a generic $F \in \operatorname{Im}\left(\Phi_{d, l}\right)$. We want to show that there exists

$$
\left(L_{1}, \ldots, L_{l}, M_{1}, \ldots, M_{l}\right) \in \Phi_{d, l}^{-1}(F)
$$

such that the linear forms define a star configuration. Define $\Delta \subset S_{1} \times \cdots \times S_{1} \times S_{d-l+1} \times$ $\cdots \times S_{d-l+1}$ as follows:

$$
\Delta=\left\{\begin{array}{l|l}
\left(L_{1}, \ldots, L_{l}, M_{1}, \ldots, M_{l}\right) & \begin{array}{l}
\text { there exists } a \neq b \neq c \text { such that } \\
L_{a}, L_{b}, L_{c} \text { are linearly dependent }
\end{array}
\end{array}\right\} .
$$

It suffices to show that $\Phi_{d, l}^{-1}(F)$ is not contained in $\Delta$ for a generic form $F \in \operatorname{Im}\left(\Phi_{d, l}\right)$. Suppose for a contradiction that $\Phi_{d, l}^{-1}(F)$ is contained in $\Delta$. Then $\Delta$ would be a component of the domain of $\Phi_{d, l}$. This is a contradiction as the latter is an irreducible variety being the product of irreducible varieties. This completes the proof.

As a consequence of the above lemma, we only need to compute $\operatorname{dim} \operatorname{Im}\left(\Phi_{d, l}\right)$. As we now show, we can compute $\operatorname{dim} \operatorname{Im}\left(\Phi_{d, l}\right)$ by the size of its tangent space. In fact, this value will equal the vector space dimension of a graded ideal in a specific degree. 
Lemma 2.8. Let $l \geq 2$ and $d \geq l-1$ be integers, and consider $l$ general linear forms $L_{1}, \ldots, L_{l}$ in $S$. Also, let $M_{1}, \ldots, M_{l} \in S_{d-l+1}$ be any homogeneous forms of degree $d-l+1$. Set

$$
\hat{L}_{i}=\prod_{j \neq i} L_{j} \text { and } \hat{L}_{i, j}=\prod_{h \notin\{i, j\}} L_{h} \text { for } i \neq j .
$$

Define the following $l$ forms of degree $d-1$ :

$$
\begin{aligned}
Q_{1} & =M_{2} \hat{L}_{1,2}+M_{3} \hat{L}_{1,3}+\cdots M_{l} \hat{L}_{1, l}=\sum_{i \neq 1} M_{i} \hat{L}_{1, i} \\
Q_{2} & =M_{1} \hat{L}_{2,1}+M_{3} \hat{L}_{2,3}+\cdots M_{l} \hat{L}_{2, l}=\sum_{i \neq 2} M_{i} \hat{L}_{2, i} \\
& \vdots \\
Q_{l} & =M_{1} \hat{L}_{l, 1}+M_{2} \hat{L}_{l, 2}+\cdots M_{l-1} \hat{L}_{l, l-1}=\sum_{i \neq l} M_{i} \hat{L}_{l, i} .
\end{aligned}
$$

With this notation, form the ideal

$$
I=\left(\hat{L}_{1}, \cdots, \hat{L}_{l}\right)+\left(Q_{1}, \ldots, Q_{l}\right)=I_{\mathbb{X}(l)}+\left(Q_{1}, \ldots, Q_{l}\right) \subseteq S .
$$

Then $I_{d}$ is the affine tangent space to $\operatorname{Im}\left(\Phi_{d, l}\right)$ at a generic point. In particular,

$$
\operatorname{dim} \mathcal{S}(d, l)=\operatorname{dim}_{k} I_{d}-1 \text {. }
$$

Proof. The statement about $\operatorname{dim} \mathcal{S}(d, l)$ follows from the first statement and the previous lemma. We need to determine the tangent space $\operatorname{Im}\left(\Phi_{d, l}\right)$ in a generic point $q=\Phi_{d, l}(p)$, where $p=\left(L_{1}, \ldots, L_{l}, M_{1}, \ldots, M_{l}\right)$. We denote with $T_{q}$ this affine tangent space.

The elements of the tangent space $T_{q}$ are obtained as

$$
\left.\frac{d}{d t}\right|_{t=0} \Phi_{d, l}\left(L_{1}+t L_{1}^{\prime}, \ldots, L_{l}+t L_{l}^{\prime}, M_{1}+t M_{1}^{\prime}, \ldots, M_{l}+t M_{l}^{\prime}\right)
$$

when we vary the forms $L_{i}^{\prime} \in S_{1}$ and $M_{i}^{\prime} \in S_{d-l+1}$. By a direct computation we see that the elements of $T_{q}$ have the form

$$
\begin{gathered}
M_{1}^{\prime} \hat{L}_{1}+\cdots+M_{l}^{\prime} \hat{L}_{l}+L_{1}^{\prime}\left(M_{2} \hat{L}_{1,2}+\cdots+M_{l} \hat{L}_{1, l}\right)+\cdots+ \\
+L_{j}^{\prime}\left(M_{1} \hat{L}_{j, 1}+\cdots+M_{l} \hat{L}_{j, l}\right)+\cdots+L_{l}^{\prime}\left(M_{1} \hat{L}_{l, 2}+\cdots+M_{l-1} \hat{L}_{l, l-1}\right),
\end{gathered}
$$

where $\hat{L}_{i}=\prod_{j \neq i} L_{j}$ and $\hat{L}_{i, j}=\prod_{h \notin\{i, j\}} L_{h}$, for $i \neq j$.

Since the $L_{i}^{\prime} \in S_{1}$ and $M_{i}^{\prime} \in S_{d-l+1}$ can be chosen freely, we obtain $I_{d}=T_{q}$.

Remark 2.9. As in [6], we can use the above lemma and appeal to upper-semicontinuity to compute $\operatorname{dim} \mathcal{S}(d, l)$ if we know a (good) upper bound on $\operatorname{dim} \mathcal{S}(d, l)$. Indeed, suppose we know that $\operatorname{dim} \mathcal{S}(d, l) \leq M$. Given $d$ and $l$ we construct the ideal $I$ as in Lemma 2.8 by choosing forms $L_{i}$ and $M_{i}$. Then we compute $\operatorname{dim}_{k} I_{d}$ using a computer algebra system. If $\operatorname{dim}_{k} I_{d}-1=M$, by upper semi-continuity of the dimension (indeed, the dimension can decrease only on a proper closed subset), we have proved $M=\operatorname{dim}_{k} I_{d}-1 \leq \operatorname{dim} \mathcal{S}(d, l) \leq$ $M$, and hence $\operatorname{dim} \mathcal{S}(d, l)=M$ for this pair $(d, l)$. We will require this technique for some small values of $d$ and $l$. 
Using our notation, it is known that the generic plane quartic does not contain a $\mathbb{X}(5)$. We call a quartic containing a $\mathbb{X}(5)$ a Lüroth quartic. These objects were classically studied; see for example [2, 8, 13, 14], and for a modern treatment [15, 16]. Of interest is the following theorem.

Theorem 2.10 ([15, Theorem 11.4]). Lüroth quartics form a hypersurface of degree 54 in the space of plane quartics.

We can now prove $\operatorname{dim} \mathcal{S}(4,5)=13$ using our techniques.

Remark 2.11. By Lemma 2.4, $\operatorname{dim} \mathcal{S}(4,5) \leq\left(\begin{array}{l}6 \\ 4\end{array}\right)-\left(\begin{array}{l}5 \\ 2\end{array}\right)+2 \cdot 5-1=14$. However, Theorem 2.10 implies that the projection map $\phi_{d, l}: \Sigma_{d, l} \rightarrow \mathbb{P} S_{d}$ is not dominant, so $\operatorname{dim} \mathcal{S}(4,5) \leq 13$.

Now consider the following five linear forms

$$
L_{1}=x_{0} ; L_{2}=x_{1} ; L_{3}=x_{2} ; L_{4}=x_{0}+x_{1}+x_{2} ; \text { and } L_{5}=x_{0}+2 x_{1}+3 x_{3} .
$$

We construct the ideal $I$ as in Lemma 2.8 where we take $M_{1}=\cdots=M_{5}=1$. Using CoCoA $\sqrt{1}$ we find that $\operatorname{dim}_{k}\left(I_{d}\right)=14$, whence $13=\operatorname{dim}_{k}\left(I_{d}\right)-1 \leq \operatorname{dim} \mathcal{S}(4,5) \leq 13$, thus giving the desired result via Remark 2.9.

Some additional remarks about computing $\operatorname{dim}_{k} I_{d}$ are given below.

Remark 2.12. Consider the ideal $I$ in Lemma 2.8. We wish to compute $\operatorname{dim}_{k} I_{d}$. Now $I=I_{\mathbb{X}(l)}+\left(Q_{1}, \ldots, Q_{l}\right) \subseteq S$. Because $d \geq l-1$, by Lemma $2.1 \operatorname{dim}_{k}\left(I_{\mathbb{X}(l)}\right)_{d}=\left(\begin{array}{c}2+d \\ 2\end{array}\right)-\left(\begin{array}{c}l \\ 2\end{array}\right)$. It then follows that to compute $\operatorname{dim}_{k} I_{d}$, it is enough to compute $\operatorname{dim}_{k}\left(\bar{Q}_{1}, \ldots, \bar{Q}_{l}\right)_{d}$ where $\left(\bar{Q}_{1}, \ldots, \bar{Q}_{l}\right)=I / I_{\mathbb{X}(l)} \subseteq S / I_{\mathbb{X}(l)}$. So we have

$$
\operatorname{dim} \mathcal{S}(d, l)=\operatorname{dim}_{k}\left(I_{d}\right)-1=\operatorname{dim}_{k}\left(\bar{Q}_{1}, \ldots, \bar{Q}_{l}\right)_{d}+\left(\begin{array}{c}
2+d \\
2
\end{array}\right)-\left(\begin{array}{l}
l \\
2
\end{array}\right)-1
$$

By Lemma 2.4, we know $\operatorname{dim} \mathcal{S}(d, l) \leq\left(\begin{array}{c}2+d \\ 2\end{array}\right)-\left(\begin{array}{l}l \\ 2\end{array}\right)+2 l-1$. So, if we can show that $\operatorname{dim}_{k}\left(\bar{Q}_{1}, \ldots, \bar{Q}_{l}\right)=2 l$ for a specific choice of $Q_{i}$ 's, then by Remark 2.9, we will in fact have the equality $\operatorname{dim} \mathcal{S}(d, l)=\left(\begin{array}{c}2+d \\ 2\end{array}\right)-\left(\begin{array}{l}l \\ 2\end{array}\right)+2 l-1$.

We will employ the following strategy in Sections 3 and 4 . After fixing some star configuration $\mathbb{X}(l)$, we identify $2 l$ points $p_{i}$ in the star configuration, and determine $2 l$ linear forms $H_{1}, \ldots, H_{2 l}$. We then construct a $2 l \times 2 l$ evaluation matrix

\begin{tabular}{c|ccccc} 
& $H_{1} Q_{1}$ & $H_{2} Q_{1}$ & $H_{3} Q_{2}$ & $\cdots$ & $H_{2 l} Q_{l}$ \\
\hline$p_{1}$ & $\delta_{1,1}$ & $\delta_{1,2}$ & $\delta_{1,3}$ & $\cdots$ & $\delta_{1,2 l}$ \\
$p_{2}$ & $\delta_{2,1}$ & $\delta_{2,2}$ & $\delta_{2,3}$ & $\cdots$ & $\delta_{2,2 l}$ \\
$p_{2}$ & $\delta_{3,1}$ & $\delta_{3,2}$ & $\delta_{3,3}$ & $\cdots$ & $\delta_{3,2 l}$ \\
$\vdots$ & & & & $\vdots$ & \\
$p_{2 l}$ & $\delta_{2 l, 1}$ & $\delta_{2 l, 2}$ & $\delta_{2 l, 3}$ & $\cdots$ & $\delta_{2 l, 2 l}$
\end{tabular}

where $\delta_{i, j}$ is the point $p_{i}$ evaluated at the degree $d$ form that indexes column $j$. If $\mathcal{M}$ denotes the resulting matrix, then $\operatorname{rk}(\mathcal{M})=\operatorname{dim}_{k}\left(\bar{Q}_{1}, \ldots, \bar{Q}_{l}\right)_{d}$ in $S / I_{\mathbb{X}(l)}$.

\footnotetext{
${ }^{1}$ For our code, see http://flash.lakeheadu.ca/ avantuyl/research/PlaneCurvesStarConfig_CGVT.html
} 
3. THE CASE $l=6$ AND $d \geq 5$

In this section we compute $\operatorname{dim} \mathcal{S}(d, 6)$ for all $d \geq 6-1=5$. We make use of the following notion: if $\mathbb{X}(l)$ is the star configuration constructed from $L_{1}, \ldots, L_{l}$, we let $p_{i, j}$ with $1 \leq i<j \leq l$ denote the point formed by intersection of $\ell_{i}$ and $\ell_{j}$. Thus $\mathbb{X}(l)=\left\{p_{i, j}=\ell_{i} \cap \ell_{j}: 1 \leq i<j \leq l\right\}$.

Theorem 3.1. For all $d \geq 5, \operatorname{dim} \mathcal{S}(d, 6)=\left(\begin{array}{c}d+2 \\ 2\end{array}\right)-4$.

Proof. We break the proof into three cases: (1) $d=5,(2) d=6$, and (3) $d \geq 7$. For all three cases, we will use the strategy outlined in Remark 2.12, thus, it suffices to construct a $12 \times 12$ evaluation matrix with maximal rank.

(1) For the case $d=5$, consider the six linear forms

$L_{1}=x_{0} ; L_{2}=x_{1} ; L_{3}=x_{2} ; L_{4}=x_{0}+x_{1}+x_{2} ; L_{5}=x_{0}+2 x_{1}+3 x_{2} ;$ and $L_{6}=x_{0}+3 x_{1}+10 x_{2}$.

When constructing the $Q_{i}$ 's, we set $M_{i}=1$ for $i=1, \ldots, 6$. We form the evaluation matrix with columns indexed by

$$
L_{2} Q_{4}, L_{1} Q_{4}, L_{3} Q_{5}, L_{2} Q_{5}, L_{1} Q_{6}, L_{3} Q_{6}, L_{6} Q_{1}, L_{3} Q_{2}, L_{6} Q_{2}, L_{6} Q_{3}, L_{4} Q_{3}, L_{5} Q_{1}
$$

and rows are index by

$$
p_{1,4}, p_{2,4}, p_{2,5}, p_{3,5}, p_{3,5}, p_{3,6}, p_{2,6}, p_{1,5}, p_{2,6}, p_{2,3}, p_{3,4}, p_{1,2} \text {. }
$$

We used CoCoA to verify that the resulting matrix has the desired rank of 12 (our code can be found on the third author's website).

(2) For the case $d=6$, we use the same $L_{i}$ 's, but when we construct the $Q_{i}$ 's, we first find a linear form $G$ that does not contain any of the points of $\mathbb{X}(6)$, and set $M_{i}=G$ for $i=1, \ldots, 6$. Again, the resulting evaluation matrix (using the same indexing for the rows and columns) has maximal rank.

(3) We now consider the case $d \geq 7$. Pick any six general linear forms $L_{1}, \ldots, L_{6}$ that form a $\mathbb{X}(6)$, and let $p_{1,2}, \ldots, p_{4,5}$ be the 15 points of $\mathbb{X}(6)$. In order to construct the $Q_{i}$ 's as defined in Lemma 2.8, we need to pick six forms $M_{1}, \ldots, M_{6}$ in $S_{d-l+1}$. Since $d \geq 7$, each $M_{i}$ will have degree at least two. We construct the $M_{i}$ 's as follows. First, we pick six linear forms in $S$ with the following properties:

- $G$ is a linear form such that the line $G=0$ does not pass through any point of $\mathbb{X}(6)$

- $G_{1}$ is a linear form such that line $G_{1}=0$ only passes through the point $p_{1,5}$ of $\mathbb{X}(6)$

- $G_{2}$ is a linear form such that the line $G_{2}=0$ only passes through the point $p_{1,2}$ of $\mathbb{X}(6)$;

- $G_{3}$ is a linear form such that the line $G_{3}=0$ only passes through the point $p_{2,6}$ of $\mathbb{X}(6)$;

- $G_{4}$ is a linear form such that the line $G_{4}=0$ only passes through the point $p_{3,4}$ of $\mathbb{X}(6)$;

- $G_{5}$ is a linear form such that the line $G_{5}=0$ only passes through the point $p_{4,6}$ of $\mathbb{X}(6)$. 
We then set

$$
\begin{array}{lll}
M_{1}=G_{1} G_{2} G^{d-l-1} & M_{2}=G_{3} G^{d-l} & M_{3}=G_{4} G^{d-l} \\
M_{4}=G^{d-l+1} & M_{5}=G^{d-l+1} & M_{6}=G_{5} G^{d-l}
\end{array}
$$

and use these $M_{i}$ 's to construct $Q_{1}, \ldots, Q_{6}$.

We now consider the $12 \times 12$ evaluation matrix (see below) whose columns and rows are also indexed as above. When determining the entries of the evaluation matrix, note that $L_{r} Q_{t}\left(p_{i, j}\right)=0$ if $i=r$, or $j=r$, or neither $i$ and $j$ equal $t$. We will also have

$$
\begin{array}{lllll}
L_{3} Q_{5}\left(p_{1,5}\right)=0 & L_{1} Q_{6}\left(p_{2,6}\right)=0 & L_{2} Q_{4}\left(p_{3,4}\right)=0 & L_{2} Q_{4}\left(p_{4,6}\right)=0 & L_{3} Q_{2}\left(p_{1,2}\right)=0 \\
L_{2} Q_{5}\left(p_{1,5}\right)=0 & L_{3} Q_{6}\left(p_{2,6}\right)=0 & L_{1} Q_{4}\left(p_{3,4}\right)=0 & L_{1} Q_{4}\left(p_{4,6}\right)=0 & L_{6} Q_{2}\left(p_{1,2}\right)=0
\end{array}
$$

This follows from our choice of $M_{i}$ 's. For example,

$$
\begin{aligned}
L_{3} Q_{5}\left(p_{1,5}\right) & =L_{3}\left(M_{1} L_{2} L_{3} L_{4} L_{6}+M_{2} L_{1} L_{3} L_{4} L_{6}+M_{3} L_{1} L_{2} L_{4} L_{6}+M_{4} L_{1} L_{2} L_{3} L_{6}+M_{6} L_{1} L_{2} L_{3} L_{4}\right)\left(p_{1,5}\right) \\
& =M_{1} L_{2} L_{3}^{2} L_{4} L_{6}\left(p_{1,5}\right)=0
\end{aligned}
$$

since $M_{1}\left(p_{1,5}\right)=0$. Again by our choice of $M_{i}^{\prime}$ 's, we have

$$
\begin{array}{lllll}
L_{2} Q_{4}\left(p_{1,4}\right) \neq 0 & L_{1} Q_{4}\left(p_{2,4}\right) \neq 0 & L_{3} Q_{5}\left(p_{2,5}\right) \neq 0 & L_{2} Q_{5}\left(p_{3,5}\right) \neq 0 & L_{1} Q_{6}\left(p_{3,6}\right) \neq 0 \\
L_{3} Q_{3}\left(p_{1,6}\right) \neq 0 & L_{4} Q_{3}\left(p_{3,6}\right) \neq 0 & L_{5} Q_{1}\left(p_{1,6}\right) \neq 0 & L_{1} Q_{6}\left(p_{4,6}\right) \neq 0 & L_{3} Q_{6}\left(p_{4,6}\right) \neq 0 \\
& L_{6} Q_{1}\left(p_{1,2}\right) \neq 0 & L_{5} Q_{1}\left(p_{1,2}\right) \neq 0 . & &
\end{array}
$$

For example,

$$
\begin{aligned}
L_{5} Q_{1}\left(p_{1,2}\right) & =L_{5}\left(M_{2} L_{3} L_{4} L_{5} L_{6}+M_{3} L_{2} L_{4} L_{5} L_{6}+M_{4} L_{2} L_{3} L_{5} L_{6}+M_{5} L_{2} L_{3} L_{4} L_{6}+M_{6} L_{2} L_{3} L_{4} L_{5}\right)\left(p_{1,2}\right) \\
& =M_{2} L_{3} L_{4} L_{5}^{2} L_{6}\left(p_{1,2}\right) \neq 0
\end{aligned}
$$

since $M_{2}$ does not vanish at $p_{1,2}$, and $p_{1,2}$ does not lie on the lines defined by $L_{3}, L_{4}, L_{5}$ or $L_{6}$.

Our evaluation matrix therefore has the form

\begin{tabular}{c|cccccccccccc} 
& $L_{2} Q_{4}$ & $L_{1} Q_{4}$ & $L_{3} Q_{5}$ & $L_{2} Q_{5}$ & $L_{1} Q_{6}$ & $L_{3} Q_{6}$ & $L_{6} Q_{1}$ & $L_{3} Q_{2}$ & $L_{6} Q_{2}$ & $L_{6} Q_{3}$ & $L_{4} Q_{3}$ & $L_{5} Q_{1}$ \\
\hline$p_{1,4}$ & $\star$ & 0 & 0 & 0 & 0 & 0 & $\square$ & 0 & 0 & 0 & 0 & $\square$ \\
$p_{2,4}$ & 0 & $\star$ & 0 & 0 & 0 & 0 & 0 & $\square$ & $\square$ & 0 & 0 & 0 \\
$p_{2,5}$ & 0 & 0 & $\star$ & 0 & 0 & 0 & 0 & 0 & $\square$ & 0 & 0 & 0 \\
$p_{3,5}$ & 0 & 0 & 0 & $\star$ & 0 & 0 & 0 & 0 & 0 & $\square$ & $\square$ & 0 \\
$p_{3,6}$ & 0 & 0 & 0 & 0 & $\star$ & 0 & 0 & 0 & 0 & 0 & $\star$ & 0 \\
$p_{1,6}$ & 0 & 0 & 0 & 0 & 0 & $\star$ & 0 & 0 & 0 & 0 & 0 & $\star$ \\
$p_{1,5}$ & 0 & 0 & 0 & 0 & 0 & 0 & $\star$ & 0 & 0 & 0 & 0 & 0 \\
$p_{2,6}$ & 0 & 0 & 0 & 0 & 0 & 0 & 0 & $\star$ & 0 & 0 & 0 & 0 \\
$p_{2,3}$ & 0 & 0 & 0 & 0 & 0 & 0 & 0 & 0 & $\star$ & $\star$ & $\star$ & 0 \\
$p_{3,4}$ & 0 & 0 & 0 & 0 & 0 & 0 & 0 & 0 & 0 & $\star$ & 0 & 0 \\
$p_{4,6}$ & 0 & 0 & 0 & 0 & $\star$ & $\star$ & 0 & 0 & 0 & 0 & 0 & 0 \\
$p_{1,2}$ & 0 & 0 & 0 & 0 & 0 & 0 & $\star$ & 0 & 0 & 0 & 0 & $\star$
\end{tabular}

where $\star$ denotes a nonzero entry and $\square$ denotes an entry which may or may not be zero. Using Gaussian elimination, we get a matrix in row echelon form (where the nonzero leading coefficients are not necessarily equal to 1 ), with zero entries below the diagonal. Consequently, the original matrix has maximal rank, as desired. 


\section{THE CASE $l>6$ AND $d \geq l-1$}

We now evaluate $\operatorname{dim} \mathcal{S}(d, l)$ for all $l \geq 6$ when $d \geq l-1$. The key idea is to pick the $l$ linear forms $L_{1}, \ldots, L_{l}$ that define $\mathbb{X}(l)$ so that the first six forms are as in Theorem 3.1 .

Theorem 4.1. Let $l \geq 7$ and $d \geq l-1$. Then $\operatorname{dim} \mathcal{S}(d, l)=\left(\begin{array}{c}d+2 \\ 2\end{array}\right)-\left(\begin{array}{l}l \\ 2\end{array}\right)+2 l-1$.

Proof. As in Theorem 3.1, it suffices to construct a $2 l \times 2 l$ evaluation matrix of rank $2 l$. Let $L_{1}, \ldots, L_{l}$ be the $l$ general linear forms that define $\mathbb{X}(l)$. If $d=l-1$ or $d=l$, we let $L_{1}, \ldots, L_{6}$ be as in Theorem 3.1. When constructing the $Q_{i}$ 's, we use the following $M_{i}$ 's:

- If $d=l-1$, let $M_{i}=1$ for $i=1, \ldots, l$.

- If $d=l$, let $M_{i}=G$, where $G$ is a linear form such that the curve $G=0$ does not contain any of the points of $\mathbb{X}(l)$.

- If $d \geq l+1$, define $M_{1}, \ldots, M_{6}$ as in Theorem 3.1, but with the added condition that each $M_{i}$ also does not vanish at any other point of $\mathbb{X}(l)$. We set $M_{7}=\cdots=$ $M_{l}=G^{d-l+1}$, where again $G$ is a linear form such that the curve $G=0$ does not contain any points of $\mathbb{X}(l)$.

When we form our evaluation matrix, we label the first twelve columns as in Theorem 3.1, and we label the remain $2 l-12$ columns with $L_{2} Q_{7}, L_{1} Q_{7}, L_{2} Q_{8}, L_{1} Q_{8}, \ldots, L_{2} Q_{l}, L_{1} Q_{l}$. We label the first twelve rows as in Theorem 3.1 and the remaining rows are labelled with $p_{1,7}, p_{2,7}, p_{1,8}, p_{2,8}, \ldots, p_{1, l}, p_{2, l}$. Our evaluation matrix then has the form:

\begin{tabular}{c|ccccc|ccccccc} 
& $L_{2} Q_{4}$ & $L_{1} Q_{4}$ & $\cdots$ & $L_{4} Q_{3}$ & $L_{5} Q_{1}$ & $L_{2} Q_{7}$ & $L_{1} Q_{7}$ & $L_{2} Q_{8}$ & $L_{1} Q_{8}$ & $\cdots$ & $L_{2} Q_{l}$ & $L_{1} Q_{l}$ \\
\hline$p_{1,4}$ & $\star$ & 0 & $\cdots$ & 0 & $\square$ & 0 & 0 & 0 & 0 & $\cdots$ & 0 & 0 \\
$p_{2,4}$ & 0 & $\star$ & $\cdots$ & 0 & 0 & 0 & 0 & 0 & 0 & $\cdots$ & 0 & 0 \\
$\vdots$ & & & $\vdots$ & & & & & & & $\vdots$ & 0 & 0 \\
$p_{4,6}$ & 0 & 0 & $\cdots$ & 0 & 0 & 0 & 0 & 0 & 0 & $\cdots$ & 0 & 0 \\
$p_{1,2}$ & 0 & 0 & $\cdots$ & 0 & $\star$ & 0 & 0 & 0 & 0 & $\cdots$ & 0 & 0 \\
\hline$p_{1,7}$ & 0 & 0 & $\cdots$ & 0 & $\square$ & $\star$ & 0 & 0 & 0 & $\cdots$ & 0 & 0 \\
$p_{2,7}$ & 0 & 0 & $\cdots$ & 0 & $\square$ & 0 & $\star$ & 0 & 0 & $\cdots$ & 0 & 0 \\
$p_{1,8}$ & 0 & 0 & $\cdots$ & 0 & $\square$ & 0 & 0 & $\star$ & 0 & $\cdots$ & 0 & 0 \\
$p_{2,8}$ & 0 & 0 & $\cdots$ & 0 & $\square$ & 0 & 0 & 0 & $\star$ & $\cdots$ & 0 & 0 \\
$\vdots$ & & & $\vdots$ & & & & & & & $\vdots$ & 0 & 0 \\
$p_{1, l}$ & 0 & 0 & $\cdots$ & 0 & $\square$ & 0 & 0 & 0 & 0 & $\cdots$ & $\star$ & 0 \\
$p_{2, l}$ & 0 & 0 & $\cdots$ & 0 & $\square$ & 0 & 0 & 0 & 0 & $\cdots$ & 0 & $\star$
\end{tabular}

where $\star$ denotes a nonzero entry and $\square$ denotes an entry which may or may not be zero.

Consider the $12 \times 12$ sub-matrix formed by the first 12 rows and 12 columns. Let $Q_{i}^{\prime}$ denote the form constructed as in Lemma 2.8 using $L_{1}, \ldots, L_{6}$ and the same $M_{i}$ 's as above. Then, for every nonzero entry in this sub-matrix, we have

$$
L_{r} Q_{t}\left(p_{i, j}\right)=L_{r} Q_{t}^{\prime} L_{7} L_{8} \cdots L_{l}\left(p_{i, j}\right)=\left[L_{r} Q_{t}^{\prime}\left(p_{i, j}\right)\right]\left[L_{7} L_{8} \cdots L_{l}\left(p_{i, j}\right)\right] .
$$

For example

$$
\begin{aligned}
L_{2} Q_{4}\left(p_{1,4}\right) & =L_{2}\left(M_{1} \hat{L}_{4,1}+M_{2} \hat{L}_{4,2}+\cdots M_{l} \hat{L}_{4, l}\right)\left(p_{1,4}\right)=L_{2} M_{1} \hat{L}_{4,1}\left(p_{1,4}\right) \\
& =L_{2}\left(M_{1} L_{2} L_{3} L_{5} L_{6} L_{7} \cdots L_{l}\right)\left(p_{1,4}\right)=\left[L_{2}\left(M_{1} L_{2} L_{3} L_{5} L_{6}\right)\left(p_{1,4}\right)\right]\left[L_{7} \cdots L_{l}\left(p_{1,4}\right)\right] .
\end{aligned}
$$


We can factor our evaluation matrix as $A B$ where

$$
A=\left[\begin{array}{cccccc}
L_{7} \cdots L_{l}\left(p_{1,4}\right) & 0 & \cdots & 0 & 0 & \\
0 & \left.L_{7} \cdots L_{l}\left(p_{2,4}\right)\right) & & 0 & 0 & \\
\vdots & & \ddots & & & 0 \\
0 & & & L_{7} \cdots L_{l}\left(p_{4,6}\right) & 0 & \\
0 & 0 & \cdots & 0 & L_{7} \cdots L_{l}\left(p_{1,2}\right) & \\
& & \mathbf{0} & & & I_{2 l-12}
\end{array}\right]
$$

where $\mathbf{0}$ denotes an appropriate sized zero matrix, and $I_{2 l-12}$ is the identity matrix, and where $B$ is the matrix given by

\begin{tabular}{c|ccccc|ccccccc} 
& $L_{2} Q_{4}^{\prime}$ & $L_{1} Q_{4}^{\prime}$ & $\cdots$ & $L_{4} Q_{3}^{\prime}$ & $L_{5} Q_{1}^{\prime}$ & $L_{2} Q_{7}$ & $L_{1} Q_{7}$ & $L_{2} Q_{8}$ & $L_{1} Q_{8}$ & $\cdots$ & $L_{2} Q_{l}$ & $L_{1} Q_{l}$ \\
\hline$p_{1,4}$ & $\star$ & 0 & $\cdots$ & 0 & $\square$ & 0 & 0 & 0 & 0 & $\cdots$ & 0 & 0 \\
$p_{2,4}$ & 0 & $\star$ & $\cdots$ & 0 & 0 & 0 & 0 & 0 & 0 & $\cdots$ & 0 & 0 \\
$\vdots$ & & & $\vdots$ & & & & & & & $\vdots$ & 0 & 0 \\
$p_{4,6}$ & 0 & 0 & $\cdots$ & 0 & 0 & 0 & 0 & 0 & 0 & $\cdots$ & 0 & 0 \\
$p_{1,2}$ & 0 & 0 & $\cdots$ & 0 & $\star$ & 0 & 0 & 0 & 0 & $\cdots$ & 0 & 0 \\
\hline$p_{1,7}$ & 0 & 0 & $\cdots$ & 0 & $\square$ & $\star$ & 0 & 0 & 0 & $\cdots$ & 0 & 0 \\
$p_{2,7}$ & 0 & 0 & $\cdots$ & 0 & $\square$ & 0 & $\star$ & 0 & 0 & $\cdots$ & 0 & 0 \\
$p_{1,8}$ & 0 & 0 & $\cdots$ & 0 & $\square$ & 0 & 0 & $\star$ & 0 & $\cdots$ & 0 & 0 \\
$p_{2,8}$ & 0 & 0 & $\cdots$ & 0 & $\square$ & 0 & 0 & 0 & $\star$ & $\cdots$ & 0 & 0 \\
$\vdots$ & & & $\vdots$ & & & & & & & $\vdots$ & 0 & 0 \\
$p_{1, l}$ & 0 & 0 & $\cdots$ & 0 & $\square$ & 0 & 0 & 0 & 0 & $\cdots$ & $\star$ & 0 \\
$p_{2, l}$ & 0 & 0 & $\cdots$ & 0 & $\square$ & 0 & 0 & 0 & 0 & $\cdots$ & 0 & $\star$
\end{tabular}

But now the matrix $B$ has the property that the $12 \times 12$ sub-matrix in the upper left hand corner is exactly the same as the matrix as in Theorem 3.1. As a result, this sub-matrix has rank 12. Furthermore, the lower $(2 l-12) \times(2 l-12)$ sub-matrix clearly has maximal rank, and so $B$ has maximal rank. Finally, since none of the points indexing the first 12 rows vanish at $L_{7}, \ldots, L_{l}$, the matrix $A$ also has maximal rank, so our original evaluation matrix has the desired rank of $2 l$.

For completeness, we now put together all the pieces to prove our main theorem.

Proof. (of Theorem 1.1) By Lemma 2.1 and Remark 2.2, $\mathcal{S}(d, l)=\emptyset$ if $d<l-1$. The value for $\operatorname{dim} \mathcal{S}(4,5)$ comes from Remark 2.11. The main theorem of [6] determines when $\operatorname{dim} \mathcal{S}(d, l)=\left(\begin{array}{c}d+2 \\ 2\end{array}\right)-1$. Theorems 3.1 and 4.1 cover the remaining cases.

\section{Concluding Remarks}

It is natural to ask whether the work of this paper can be generalized to star configurations set of points $\mathbb{X}(l)$ in $\mathbb{P}^{n}$; see [5] for more on this. Indeed, let $\Sigma_{n, d, l}$ be the incidence correspondence

$$
\Sigma_{n, d, l}=\{(\mathcal{H}, \mathbb{X}(l)): \mathcal{H} \supseteq \mathbb{X}(l)\} \subseteq \mathbb{P} S_{d} \times \mathcal{D}_{l} .
$$

where now $S=k\left[x_{0}, \ldots, x_{n}\right]$ and $\mathcal{D}_{l} \subseteq \check{\mathbb{P}}^{n} \times \cdots \times \check{\mathbb{P}}^{n}$ ( $l$ times). Letting $\phi_{n, d, l}: \Sigma_{n, d, l} \rightarrow \mathbb{P} S_{d}$ denote the natural projection map, we wish to compute the dimension of the corresponding locus, that is, $\mathcal{S}(d, l, n)=\phi_{n, d, l}\left(\Sigma_{n, d, l}\right)$. 
The proofs of Section 2 extend naturally to this case, thus giving us the upper bound

$$
\operatorname{dim} \mathcal{S}(d, l, n) \leq \min \left\{\left(\begin{array}{c}
d+n \\
n
\end{array}\right)-1,\left(\begin{array}{c}
d+n \\
n
\end{array}\right)-\left(\begin{array}{c}
l \\
n
\end{array}\right)+n l-1\right\} \text { for all } d \geq l-1
$$

Computer experiments suggest that this inequality is an equality for all $d \geq l-1$ with

$n \geq 3$. The results of [5] already verifies part of this claim when the minimum is $\left(\begin{array}{c}d+n \\ n\end{array}\right)-1$. We expect that the approach used in this paper will verify this question; however, the difficultly is now finding the correct evaluation matrix and determining its rank.

As an interesting aside, if this equality holds, this would imply that the Lüroth case is the only time $\operatorname{dim} \mathcal{S}(d, l, n)$ is not the expected value. Also notice that the case of Lüroth quartic is the only one in the plane for which the locus of star configurations is an hypersurface, and it is hence defined by a single equation. Moreover, the locus of a star configurations is never zero dimensional.

\section{REFERENCES}

[1] J. Ahn, Y.S. Shin, The minimal free resolution of a star-configuration in $\mathbb{P}^{n}$ and the Weak Lefschetz Property. J. Korean Math. Soc. 49 (2012), no. 2, 405-417.

[2] W. Barth, Moduli of vector bundles on the projective plane. Invent. Math. 42 (1977), 63-92.

[3] C. Bocci, S. Cooper, B. Harbourne, Containment results for ideals of various configurations of points in $\mathbb{P}^{N}$. J. Pure Appl. Algebra 218 (2014), no. 1, 65-75.

[4] C. Bocci, B. Harbourne, Comparing Powers and Symbolic Powers of Ideals. J. Algebraic Geom. 19 (2010), no. 3, 399-417

[5] E. Carlini, E. Guardo, A. Van Tuyl, Star configurations on generic hypersurfaces. J. Algebra 407 (2014), 1-20.

[6] E. Carlini, A. Van Tuyl, Star configuration points and generic plane curves. Proc. Amer. Math. Soc. 139 (2011), no. 12, 4181-4192.

[7] CoCoATeam, CoCoA: a system for doing Computations in Commutative Algebra. Available at http://cocoa.dima.unige.it

[8] I.V. Dolgachev, Classical Algebraic Geometry: a modern view. Cambridge Univ. Press (2012).

[9] G. Ellingsrud, J. Le Potier, S.A. Strømme, Some Donaldson invariants of CP $P^{2}$. Moduli of vector bundles (Sanda, 1994; Kyoto, 1994), 33-38, Lecture Notes in Pure and Appl. Math., 179, Dekker, New York, 1996.

[10] G. Fatabbi, B. Harbourne, A. Lorenzini, Inclics, galaxies, star configurations and Waldschmidt constants. Preprint (2013). arXiv:1304.2217v2

[11] A.V. Geramita, B. Harbourne, J. Migliore, Star configurations in $\mathbb{P}^{n}$. J. Algebra 376 (2013) $279-299$.

[12] A.V. Geramita, J. Migliore, L. Sabourin, The first infinitesimal neighborhood of a linear configuration of points in $\mathbb{P}^{2}$. J. Algebra 298 (2006), no. 2, 563-611.

[13] J. Lüroth, Einige Eigenschaften einer gewissen Gattung von Curven vierter Ordnung. Math. Ann. 1 (1869), no. 1, 37-53.

[14] F. Morley, On the Lüroth Quartic Curve. Amer. J. Math. 41 (1919), no. 4, 279-282.

[15] G. Ottaviani, E. Sernesi, On the hypersurface of Lüroth quartics. Michigan Math. J. 59 (2010), no. $2,365-394$.

[16] G. Ottaviani, E. Sernesi, On singular Lüroth quartics. Science China Mathematics 54 (2011), no. 8, 1757-1766.

[17] J.P. Park, Y.S. Shin, The minimal free resolution of a star-configuration in $\mathbb{P}^{n}$. (2014) To appear J. Pure Appl. Algebra. arXiv:1404.4724v1

[18] Y.S. Shin, Some applications of the union of star-configurations in $\mathbb{P}^{n}$. J. Chungcheong Math. Soc. 24 (2011), no. 4, 807-824. 
[19] Ş. Tohăneanu, On the de Boer-Pellikaan method for computing minimum distance. J. Symbolic Comput. 45 (2010), no. 10, 965-974.

(E. Carlini) Disma, Department of Mathematical Sciences, Poltiecnico di Torino, Turin, TALY

E-mail address: enrico.carlini@polito.it

(E. Carlini) School of Mathematical Sciences, Monash University, Clayton, Australia

E-mail address: enrico.carlini@monash.edu

(E. Guardo) Dipartimento di Matematica, Universita di Catania, Catania, Italy

E-mail address: guardo@dmi.unict.it

(A. Van Tuyl) Department of Mathematical Sciences, Lakehead University, Thunder BAY, ON, CANAdA, P7B 5E1

E-mail address: avantuyl@lakeheadu.ca 